Please quote as: Köbler, F.; Goswami, S.; Koene, P.; Leimeister, J. M. \& Krcmar, H. (2011): Using NFriendConnector to Extend Facebook to the Real World. In: Wirtschaftsinformatik Konferenz (WI) 2011, Zürich, Schweiz. 
Association for Information Systems AIS Electronic Library (AISeL)

Wirtschaftinformatik Proceedings 2011

Wirtschaftinformatik

$1-1-2011$

\section{Using NFriendConnector to Extend Facebook to the Real World}

Felix Köbler

Technische Universität München, koebler@in.tum.de

Suparna Goswami

Technische Universität München, goswami@in.tum.de

Philip Koene

Technische Universität München, koene@in.tum.de

Jan Marco Leimeister

Universität Kassel, leimeister@uni-kassel.de

Helmut Krcmar

Technical Universität Munchen, krcmar@in.tum.de

\section{Recommended Citation}

Köbler, Felix; Goswami, Suparna; Koene, Philip; Leimeister, Jan Marco; and Krcmar, Helmut, "Using NFriendConnector to Extend Facebook to the Real World" (2011). Wirtschaftinformatik Proceedings 2011. Paper 27.

http://aisel.aisnet.org/wi2011/27

This material is brought to you by the Wirtschaftinformatik at AIS Electronic Library (AISeL). It has been accepted for inclusion in Wirtschaftinformatik Proceedings 2011 by an authorized administrator of AIS Electronic Library (AISeL). For more information, please contact elibrary@aisnet.org. 


\section{Using NFriendConnector to Extend Facebook to the Real World}

\author{
Felix Köbler \\ Lehrstuhl für Wirtschaftsinformatik \\ Technische Universität München \\ Boltzmannstr. 3 \\ 85748 Garching \\ koebler@in.tum.de
}

\author{
Suparna Goswami \\ Lehrstuhl für Wirtschaftsinformatik \\ Technische Universität München \\ Boltzmannstr. 3 \\ 85748 Garching \\ goswami@in.tum.de
}

\author{
Philip Koene \\ Lehrstuhl für Wirtschaftsinformatik \\ Technische Universität München \\ Boltzmannstr. 3 \\ 85748 Garching \\ koene@in.tum.de
}

\author{
Jan Marco Leimeister \\ Fachgebiet Wirtschaftsinformatik \\ Universität Kassel \\ Nora-Platiel-Str. 4 \\ 34127 Kassel \\ leimeister@uni-kassel.de
}

\author{
Helmut Krcmar \\ Lehrstuhl für Wirtschaftsinformatik \\ Technische Universität München \\ Boltzmannstr. 3 \\ 85748 Garching \\ krcmar@in.tum.de
}

\begin{abstract}
This study presents a novel approach towards establishing online social connections using Facebook and NFC-enabled mobile phones. There is increasing convergence between users' real life social networks and their online social networks, with online connections following actual social acquaintance and interactions. Accordingly there is a need to provide users with means of accessing and establishing online connections in social networking platforms such as Facebook as and when they interact with other people in their real lives. The NFriendConnector is a prototype application which fulfills this functionality. This paper describes the design and development of the prototype application. The expectation confirmation theory is used to analyze the extent to which the NFriendConnector fulfills this inherent need among users and how this influences their intention to adopt and use the prototype. The proposed research model is tested in an experimental setup.
\end{abstract}

\section{Keywords}

Social networking, mobile prototype design, mobile user interaction, expectation confirmation theory, laboratory experiment

\section{INTRODUCTION}

Online social networking has witnessed explosive growth over the past few years. This is evidenced by a nearly ten-fold growth in the number of active users on Facebook from 30 million in July 2007 to about 250 million in August 2009 [17]. Users may have various motivations for joining the different online social networking sites such as LinkedIn, Friendster, MySpace and Facebook, but the primary activity on these sites is that of forming social connections with other users. These connections can lead to potential benefits such as facilitating different tasks within the group, reducing misbehavior, creating online communities of support and generating different types of social capital $[11,13]$.

Significant research has tried to understand and categorize what motivates people to participate in various online social networking sites. Findings suggest that in previous generations of online communities, relationships were typically initiated online based on shared interests, and were likely to transcend into users' offline worlds [4, 17, 18], often resulting in face-to-face meetings. However, in the case of Facebook, users' main motivation is often to intensify and solidify relationships that started offline [10]. Accordingly, it has been proposed that people use Facebook more for the purpose of "social searching" (which refers to looking up offline contacts and acquaintances, finding out more about them, and connecting to them) as opposed to "social browsing" (which refers to finding people or groups online in order to establish connection with them) [10]. For this group of users, i.e., the social searchers, while Facebook provides the means for maintaining and strengthening of the social ties that were initially established in offline settings, users have to spend significant effort to map their real life ties in Facebook.

Facebook connections are typically established by sending a friend request. Therefore, in order to establish a connection with a person one has met in some kind of offline social setting such as in a common class, at a party, or in a conference, one has to go back to their Facebook account and search for this other person's profile. Once found, a request to become friends has to be sent to the other person, which then has to be accepted by him/her. To establish connections in this form, the person initiating the $10^{\text {th }}$ International Conference on Wirtschaftsinformatik, $16^{\text {th }}-18^{\text {th }}$ February 2011, Zurich, Switzerland 
connection has to incur certain costs in the form of having to search for the other person's profile, waiting for the other person to respond, and above all having access to a computer terminal with Internet connectivity. Thus there is still a significant gap between the initiation of contact in a real life social setting and that in an online social network.

The NFriendConnector prototype intends to fill this gap. Given the ubiquitousness of mobile phone usage in today's world, we believe that mobile phones have a significant potential of lowering the burden involved in establishing social networking connections, especially for people who frequently use Facebook to establish online connections with their offline contacts. Once the social networking profile information is present on mobile phones, it can further enrich the actual face-to-face interaction experience by enabling users to view, share and match information regarding themselves and others.

This paper presents the NFriendConnector application prototype and describes different aspects of its design and development. The prototype integrates a number of functions from the Facebook platform into a Near Field Communication (NFC) enabled mobile device, by making use of Facebook's Application Programming Interface (API) and Facebook users' profile data. It enables users to map their offline interactions as and when they take place, into their Facebook profiles and also experience a more interesting social interaction complemented by their Facebook profile information, since it allows them to share and compare it. The NFriendConnector prototype application additionally allows them to access other Facebook functionalities such as posting a new status message. Users' perceptions regarding the prototype and their intention to adopt and use it are assessed in a laboratory experiment using the expectation confirmation theory as the theoretical framework. Contributions from this research and its implications are discussed.

\section{PRIOR WORK ON MOBILE SOCIAL NETWORKING}

The widespread popularity of online social networking has resulted in researchers and system designers investigating various issues related to Internet-enabled social networking, and mechanisms for designing applications and interfaces that make online social networking more meaningful and attractive to its end-users. Accordingly, there is an emerging stream of research that has started investigating the role of mobile devices such as mobile phones, in online social networking. This is evidenced by a number of research projects targeting various aspects of mobile social networking and prototype development efforts. It is likely that commercial applications will follow suit. In fact, analysts predict that mobile social networking users are likely to make up around $18 \%$ of all mobile users, the equivalent of 950 million users worldwide by 2012 [3].

Existing research on mobile social networking explore the usage of Bluetooth, GPS, Wi-Fi and similar technologies for proximity and location detection, often called "Social Proximity Applications" [19]. One of the first projects to explore these aspects of mobile social networking was MIT's "Social Serendipity" [6], followed by others like "Dodgeball” [24] and "MobiClique" [21]. Other applications such as "DigiDress" [19] and "Sensor" [20] differentiate on certain aspects of "Social Serendipity", but share the same motivations and basic design principles. The essential idea behind these projects is to inform members with a mobile social network regarding the location and proximity of existing acquaintances in this network or other network users with whom their profile data has a match, so that they may become new acquaintances if they want to. This is achieved by a periodic Bluetooth scan of the environment and reporting all new discovered Bluetooth IDs to a central server, which then detects if the ID is part of the mobile social network and checks the corresponding profile for connections or a positive profile match. Apart from the detection of an acquaintances' location and proximity, these prototypes usually also support basic communication functions like message transfer [6, 21, 24]. Most of these prototype systems however, are based on small, proprietary social networks, with only a selected few like "MobiClique" and "Cityware" leveraging the large user base of existing online social networks $[9,21]$, as is done in the case of NFriendConnector and Facebook.

An important concern regarding mobile social networking, especially for applications making use of Bluetooth sensing is that of privacy. The implications of a user's name and details being made available to a stranger without the user's knowledge [6] or tracking user's daily routines and preferences through their location, as is done in the "Cityware" [9] mobile social network are likely to raise perceived threats towards privacy in the potential client base. In contrast to the above described mobile social networking application prototypes, the NFriendConnector does not make use of physical proximity, location or other sensory data for automatic profile matching or generation of other information. The NFriendConnector enables seamless integration of users' offline social interactions with their online social networks using a mobile phone. With NFriendConnector an interaction and exchange of information between users has to be initiated by the users themselves by establishing a near physical contact between the two mobile devices on which the NFriendConnector is running, rather than the system doing things in the background without the users' active participation or knowledge. Since the user has full control over the exchange of information that happens with other users, the NFriendConnector overcomes the privacy concerns that are associated with other mobile social networking applications.

\section{NFRIENDCONNECTOR PROTOTYPE APPLICATION}

The NFriendConnector prototype makes use of Near Field Technology (NFC) and the Representational State Transfer (REST) application programming interface (API) provided by Facebook along with the profile data of registered Facebook users. NFC is a short range high frequency wireless communication technology that allows data exchange between devices that are about 4 inches apart [18]. It is a simple extension of the ISO/IEC 14443 [7] proximity-card standard (such as contactless card, Radio-Frequency Identification (RFID), etc.) that combines the smart card and the reader into the same device. NFC is primarily aimed for being used in mobile phones. The application environment for the prototype builds on cellular phones equipped with NFC-reader devices, mobile Internet broadband connectivity enabled through Enhanced Data Rates for GSM Evolution (EDGE) or Universal Mobile Telecommunications System (UMTS) technologies and sufficient display measurements. 


\subsection{Use Case}

The simplest use case for the prototype is as follows: two individuals who are Facebook users meet in real-life in a club, a café, a common course, a conference, or some similar setting where social interaction takes place between them. Both individuals possess NFC-enabled mobile phones (as described above) with mobile Internet connectivity and the NFriendConnector application installed. The application enables them to exchange their Facebook profiles over the NFC-interface by establishing a physical contact between their cell phones. This allows them to view and compare each other's Facebook profiles. Based on this interaction, the two individuals discover common interests and a willingness to maintain the newly developed contact and keep in touch. NFriendConnector allows them now to establish a Facebook friend connection, and therefore map each other onto their existing Facebook network, and also to generate a status message.

\subsection{Prototype Navigation}

The NFriendConnector application prototype can be run in the stand-alone mode (without an active mobile Internet connection) and the connected mode (with and active mobile Internet connection). Accordingly, the features provided by the prototype are classified into client-enabled and connection-enabled. Clientenabled features can be executed in the stand-alone mode, the application accesses Facebook data which has been previously stored on the mobile device. Connection-enabled features can only be executed in the connected mode. It should be noted that the client-enabled features can also be accessed in the connected mode. The user can start the application in either of these modes and then later switch from one mode to the other if so required. The application starts with a splash screen which leads the user to the "Mode options" menu as displayed in Figure 1.
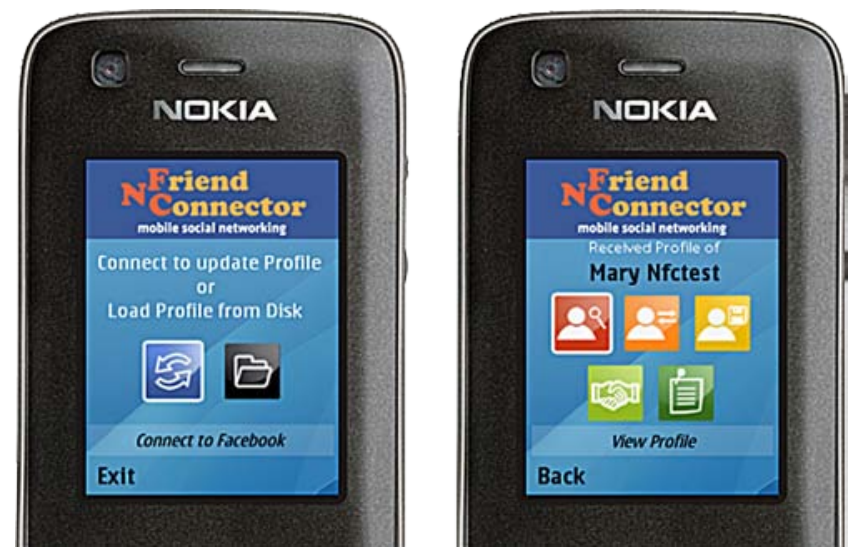

Figure 1: "Mode Options" menu (left) and "Profile Options" menu (right) of NFriendConnector

For both connected and stand-alone modes, the user can initiate a NFC-communication by touching another NFC-enabled mobile phone on which the NFriendConnector is also running. This will present the user with the "Profile options" menu (as in Figure 1) to access more features described below. Table 1 outlines the platform requirements, modes and features of the prototype.
Table 1: Requirements, Modes and Features of NFriendConnector

\begin{tabular}{|c|c|c|}
\hline \multicolumn{3}{|c|}{ NFriendConnector } \\
\hline \multirow[t]{3}{*}{ Requirements } & \multicolumn{2}{|c|}{ NFC-enabled mobile device } \\
\hline & \multicolumn{2}{|c|}{$\begin{array}{l}\text { Existing Facebook account with profile } \\
\text { information }\end{array}$} \\
\hline & & $\begin{array}{l}\text { Available mobile Internet } \\
\text { connection }\end{array}$ \\
\hline Modes & Stand-alone & Connected \\
\hline \multirow[t]{5}{*}{ Features } & \multicolumn{2}{|c|}{ View Profile } \\
\hline & \multicolumn{2}{|c|}{ Match Profile } \\
\hline & \multicolumn{2}{|c|}{ Save Profile } \\
\hline & & Add as friend \\
\hline & & Make status message \\
\hline
\end{tabular}

\subsection{Client-enabled Features}

\subsubsection{View Profile}

The "View Profile" feature displays Facebook profile data of the other individual, with whom the social interaction is taking place. The mobile phones belonging to the two Facebook users exchange the profile data through a successfully established NFCconnection. For the purpose of the prototype, only the profile picture, profile name, and the Facebook profile fields hometown, interests, movies and music are displayed as a scroll down /side scrolling list (as shown in Figure 2). It is however technically feasible to display all existing profile fields.
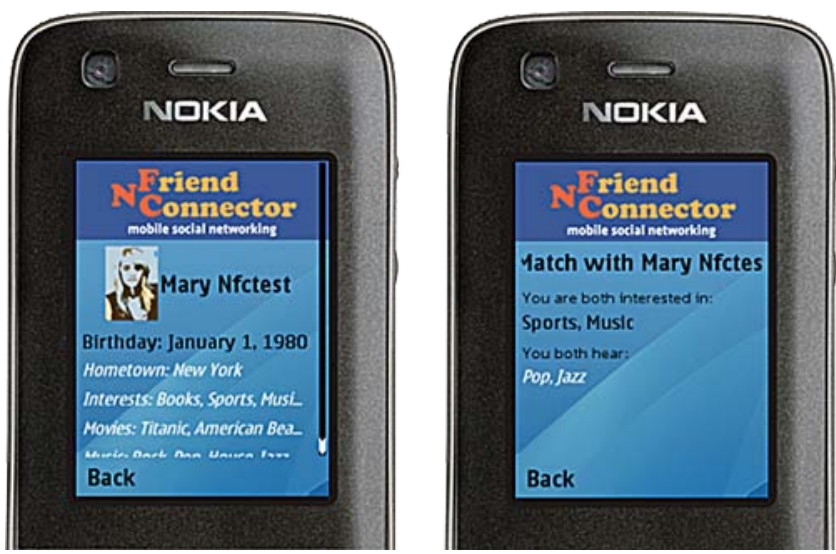

Figure 2: "View Profile” (left) and "Match Profile” (right) functionality of NFriendConnector

\subsubsection{Save Profile}

With the "Save Profile" feature, the user is able to save the Facebook profile data of the other person with whom an exchange of profile information has just happened. This allows the user to 
build an individual contact list of Facebook users on his/her mobile phone that can later be browsed even without an active mobile Internet connection.

\subsubsection{Match Profile}

The "Match Profile" feature makes use of the users' own Facebook profile data stored in the mobile phone, and the newly obtained profile information of the other person to notify if there is a match of interest, dislikes, hobbies, etc. These could be partner-/relationship-seeking, hobby-seeking (e.g., soccer club), or other matching configurations, devised by the user. For the purposes of the prototype application all existing profile fields were matched (as shown in Figure 2). It is also possible to set threshold configurations of certain variables within the profile data (such as matching relationship status, distance of home towns, count of matching interests, etc.) and calculate an output that signals the user of a positive or a negative match. This option is yet to be implemented in the NFriendConnector prototype.

\subsection{Connection-enabled Features}

The following features can only be used when the NFriendConnector is logged into Facebook through a constant mobile Internet connection.

\subsubsection{Add friend}

This feature uses the unique Facebook profile ID of the other individual to identify the individual on the Facebook platform and visit that individual's Facebook profile via the mobile web browser installed on the cell phone. The mobile web browser can be launched directly from the NFriendConnector prototype. This provides users with quick access to the Facebook function "add as a friend" in a real life social setting (Figure 3). After executing the desired Facebook functions (such as making a friend-connection request) the user can simply close the mobile web browser to return to the NFriendConnector prototype application.
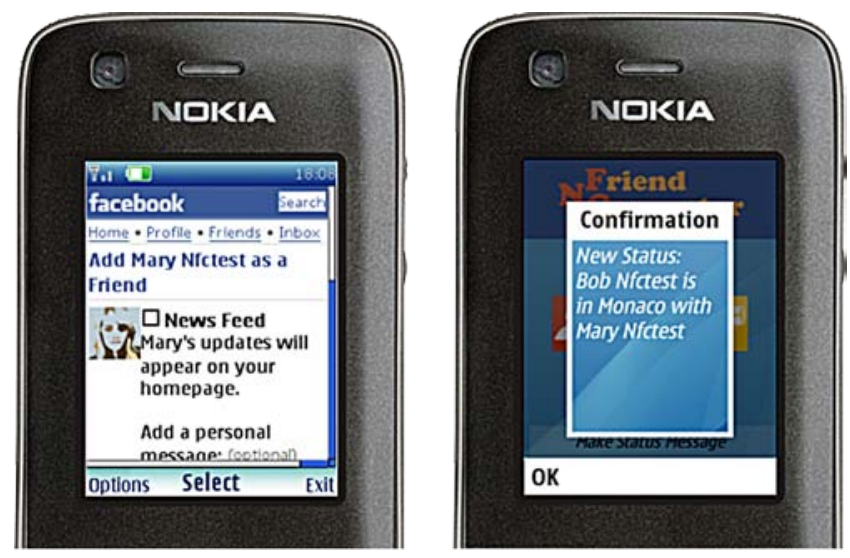

Figure 3: "Add friend" (left) and "Make status message" (right) functionality of NFriendConnector

\subsubsection{Make status message}

The NFriendConnector prototype enables users to generate automated status messages that get displayed on their respective
Facebook profile status wall. For the purposes of the prototype application, a simple example for such an automated status message is implemented into NFriendConnector. For instance the message states " $<$ individual1 $>$ is in $<$ semantic location $>$ with <individual2>" (as seen in Figure 3). The semantic location (in this case "Monaco, France") can be obtained by the GPS receiver or cell identification mechanism of the mobile phone.

The user could also define a current task (e.g., "studying”), which is then mapped onto the status message: " $<$ individual $1>$ is studying with <individual2>” or type in the status message directly through the keyboard of the cell phone. Furthermore, the automated status messages could be enriched by location-based information retrieved from the Global Positioning System (GPS) receiver in combination with other applications such as Google Maps [12].

\subsection{Implementation Details}

For the purpose of this study, the NFriendConnector is implemented on the Nokia 6212 classic NFC-enabled mobile phone [20]. It is implemented as a Java J2ME midlet that can facilitate communication with the mobile phone on one hand, and gain access to the functions and data of Facebook on the other hand. The communication with the Facebook platform is done by sending and interpreting RESTful HTTP-requests over the mobile Internet connection to the Facebook API REST server [21]. The NFC-reader device of the Nokia 6212 phone is controlled by the J2ME midlet through an API provided by Nokia [1].

Two different communication patterns are possible in the NFriendConnector prototype. Users can download and store their Facebook profile data directly in their mobile phones. Upon contact with another mobile phone on which the NFriendConnector is running, this data can be sent through the NFC-interface to the other phone. Following this exchange, the "View Profile”, "Save Profile" and "Match Profile" features can be executed. The advantage of this form of communication is that no mobile Internet connectivity is required for executing the above features. The disadvantage is the possibly large traffic over the NFC-interface, especially when profile pictures are transferred. Also the Facebook profile data on the mobile phone gets outdated without regular updates.

In the other conceivable communication routine, only the unique Facebook user ID is transferred over the NFC-interface. The rest of the profile data is then downloaded on a need basis for executing certain features. This method guarantees up-to-date profile data and low data traffic sent via the NFC-interface, however it also requires an active mobile Internet connection.

For the purpose of the prototype, a balance between the two approaches was chosen (refer to Figure 4). The user can download and update his locally stored Facebook profile upon application startup by logging into Facebook through the application. Only the information fields of the profile data, along with the unique Facebook user ID are stored on the device. This entire information is then exchanged as an XML file through the established NFCconnection with another mobile phone. Multimedia content of the profile (such as profile picture) is stored locally on an user's own mobile phone but not transmitted through the NFC-interface, in order to avoid very high NFC traffic and lengthening of the exchange process. If needed, multimedia content can be 
downloaded by the receiving device via a mobile Internet connection.

Facebook's REST API server provides access to profile data without using a browser interface. Facebook method calls can be made over the (mobile) Internet connection by sending HTTP GET or POST requests to this REST server. Most of the features of the NFriendConnector prototype are implemented in this manner.

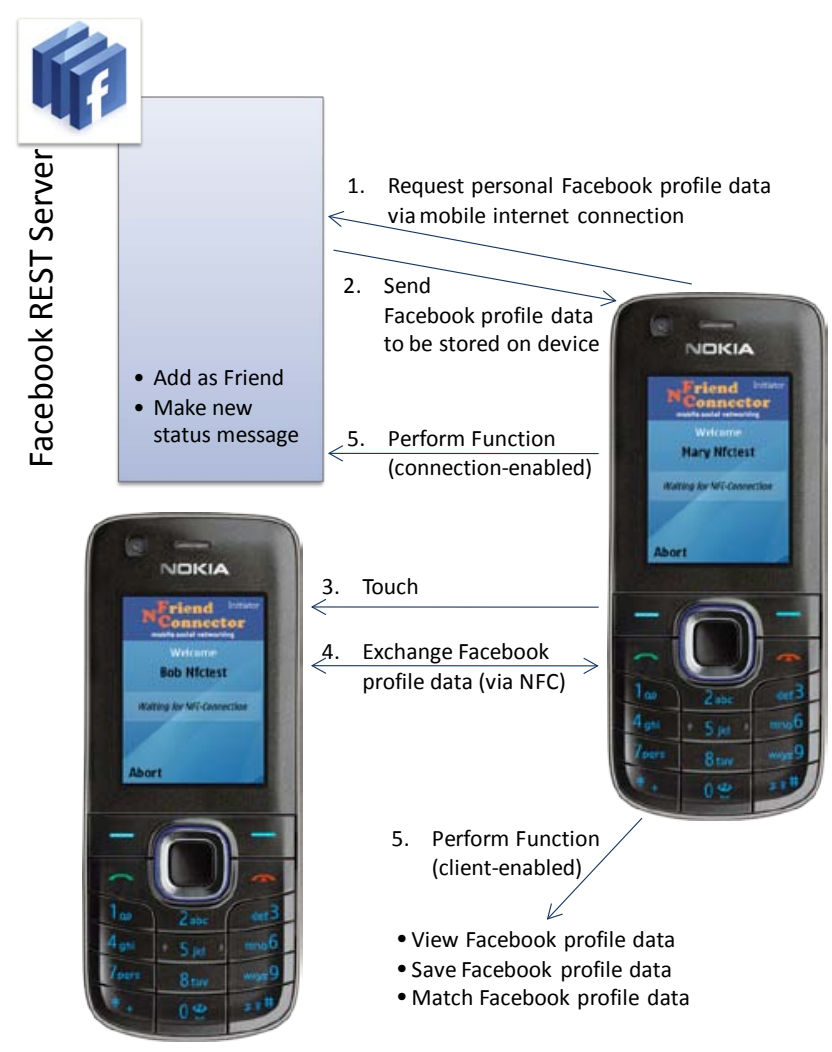

Figure 4: NFriendConnector communication routine

However, certain Facebook functionality such as logging in, or adding a friend cannot be accessed via the Facebook REST API server [21]. For executing these features, the NFriendConnector automatically opens a web browser and logs the user through the Facebook web interface from the J2ME midlet. The mobile web browser can be automatically set to a specific page, for instance the confirmation page for sending a friend request. The other user can therefore, quickly confirm the friend request and return to the NFriendConnector application by closing the mobile browser. This round-about way of accessing certain Facebook functions through a J2ME midlet is due to poor support for mobile devices currently provided by Facebook APIs. This is however, likely to change in the near future due to recent Facebook initiatives like "Facebook Platform for Mobile" [22].

\section{PROTOTYPE EVALUATION}

Previous research has indicated that in Facebook, users place a higher priority on being able to establish connections with people they have met in offline settings, than in establishing purely online connections. However, currently Facebook does not provide the functionality that supports this particular pattern of usage. The NFriendConnector addresses this by enabling users to establish a Facebook connection immediately after meeting someone of interest, provided they have an NFC-enabled mobile phone. It is expected that a prototype that satisfies this need of the users will result in increased satisfaction. The extent, to which users find the functionality provided by the NFriendConnector useful and are satisfied with it are examined in an experimental setup. The other purpose of the experiment is to assess the extent to which users are willing to adopt the prototype.

\subsection{Theoretical Basis - Expectation Confirmation}

We draw on the expectation-confirmation theory (ECT) [15] in general and the expectation confirmation model [2] in particular to explain how a confirmation of users' expectations from the system influences their satisfaction with the system. Satisfaction with an information system and intention to use it are recognized indicators of users' experience of using the system. According to ECT (Figure 5), consumers form an initial expectation regarding a particular product or service before using it. After initial consumption they assess the perceived performance based on their original expectation to determine the extent to which their expectation is confirmed. Confirmation determines satisfaction, which in turn determines repurchase or reuse intention. While initial expectation is formed prior to consumption (in time $t_{1}$ ), assessment of perceived performance, confirmation, satisfaction and repurchase intention are post-consumption variables (measured during time $\mathrm{t}_{2}$ ).

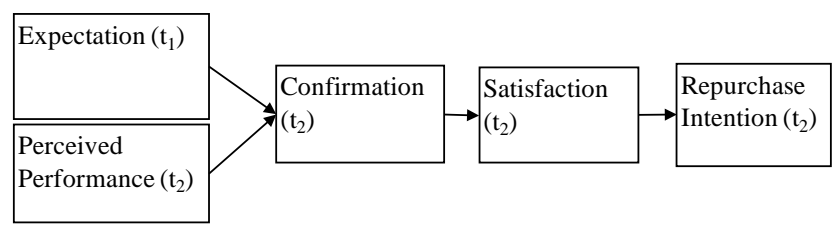

Figure 5: Expectation-confirmation theory

ECT has been used and to analyze repurchase decisions [16, 23], and more recently, to study information technology continuance behavior $[2,14]$. The underlying reasoning is that users' decisions to continue using a system is similar to that of consumers' repurchase decisions. Accordingly, the expectation confirmation model (ECM) was proposed [2] by adapting the ECT in the context of technology use and continuance. The theory was extended by drawing from other theories of technology acceptance and use such as the technology acceptance model (TAM) [5]. Instead of examining both pre- and post- use variables, only post-acceptance variables are considered. Post-use expectation is represented by perceived usefulness of the system.

\subsection{Research Model and Hypotheses}

We apply ECT as the theoretical framework to assess users' experience of using the NFriendConnector because it predicts users' intention to use a system based on their satisfaction after initial usage. Given the setting where users come to know of the prototype and use it for the first time during the course of the 
study, we are only interested in examining the post-use variables. This is because while users might have some pre-formed notions or expectations regarding the system before they actually use it for the first time, these expectations are likely to become more defined and concrete only after experiencing and using the prototype.

According to the ECT, confirmation of expectations results in satisfaction. Users evaluate their use experience based on the extent to which their expectations are confirmed and this results in them being satisfied or dissatisfied with the system. Satisfaction is an evaluative response to system use that can be either positive or negative or indifferent [2]. Therefore we hypothesize:

H1. Users' extent of confirmation is positively associated with their satisfaction of NFriendConnector use.

Users may or may not have some initial expectation regarding what the system does and how it can help them in performing their task. However, after having used the system they will form their ex-post expectation regarding the system based on its performance. Perceived usefulness [5] is used to reflect ex-post expectation in this study. Users will form their beliefs regarding the usefulness of the system by combining their evaluation vis-àvis their initial expectation of the system. The extent to which their pre-use expectations from the system are confirmed after using it will determine the extent to which they perceive the system as being useful. Therefore, we hypothesize:

H2. Users' extent of confirmation is positively associated with perceived usefulness of the NFriendConnector.

Perceived usefulness reflects a belief regarding the usefulness of the system and, beliefs typically result in affect. Satisfaction is an affect that has been found to be significantly related to perceived usefulness in the context of information systems use and continuance [2]. Since trying the NFriendConnector is an instance of information system use that will result in beliefs and subsequent affects we hypothesize:

H3. Perceived usefulness is positively associated with users' satisfaction of NFriendConnector use.

Satisfaction plays an important role in users' decision to repurchase (in consumer behavior) or continue using (in information system continuance). In the context of this study, users encounter the prototype for the first time and therefore their first usage determines the formation of the affect - satisfaction (or the lack of it). Satisfaction has already been validated as a significant predictor of intention to use in TAM [5] and various TAM-based studies (e.g., [5, 8]). Therefore, we hypothesize:

H4. Users' satisfaction with the NFriendConnector is positively associated with their intention to use it.

Previous research has shown that perceived usefulness is a salient belief that influences information system acceptance behaviors or behavioral intentions across a broad range of end-user technologies [5, 8]. Therefore, other than the relationship between perceived usefulness and satisfaction, we also hypothesize a direct link between perceived usefulness and the intention to use the NFriendConnector:

H5. Perceived usefulness is positively associated with the intention to use the NFriendConnector.
Figure 6 represents the research model for the study. All the variables in our study are measured ex-post, i.e., after users have already used the system.

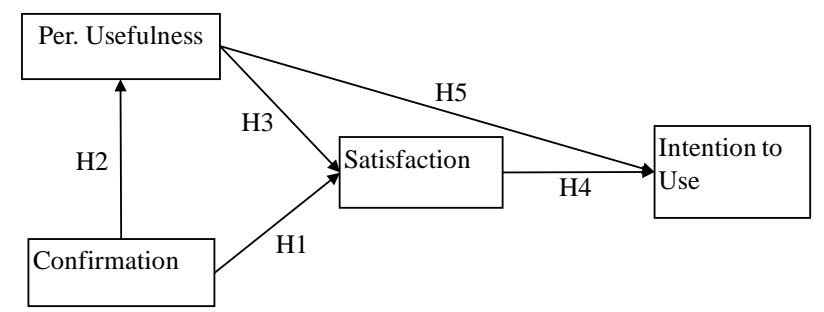

Figure 6: Research Model

\subsection{Experiment}

An experimental methodology was chosen to test the hypothesized relationships and therefore assess the usability of the NFriendConnector prototype. For this research, laboratory experiment is an appropriate methodology as it allowed us to control factors that are extraneous to the variables of interest. For instance, environmental factors were controlled for by holding the experiment in a common laboratory and using the same NFCenabled mobile devices (same make and model).

Student volunteers registered in the under-graduate or graduate programs in a large German university were recruited as experimental subjects. The experiment was held over multiple sessions in a designated laboratory. Each experimental session lasted for about 30 minutes. A standard protocol was followed for all sessions. Subjects were provided a brief introductory description about the NFriendConnector prototype and its various features, and the supporting use case. They were then given a demonstration of how they could use the prototype. Following the demonstration, they were provided with NFC-enabled mobile phones which had the prototype installed in it and were asked to evaluate the prototype. Subjects were paired and asked to use NFriendConnector for establishing Facebook connections with each other. The laboratory also had a personal computer terminal, where subjects could login to Facebook and see that the connection had actually been established. Some dummy Facebook accounts were created and subjects were asked to work with these accounts, rather than their own Facebook accounts, to ensure that all subjects worked with similar kinds of Facebook profiles.

Each subject was required to fill two short questionnaires measuring the variables of interest. The first questionnaire had to be filled at the beginning of the experiment before the subjects were introduced to the prototype, and mainly measured demographic variables such as age, educational level, and whether they were users of Facebook or not. The second questionnaire had to be filled after the subjects had used the NFriendConnector prototype. The second questionnaire measured the experimental variables such as Perceived Usefulness, Satisfaction, etc.

Validated instruments from previous research were used to measure the different variables (Table 2). Where necessary, the measurement items were modified to suit the context of this study. All items were measured using 5-point Likert scales, with scale anchors varying from 'Strongly Disagree' to 'Strongly Agree'. For satisfaction, users responded on four different scale anchors: 
Very Dissatisfied - Very Satisfied, Very Displeased - Very Pleased, Very Frustrated - Very Contended, Absolutely Terrible Absolutely Delighted.

Table 2: Measurement Instrument

\begin{tabular}{|c|c|}
\hline $\begin{array}{l}\text { Variable } \\
\text { (Source) }\end{array}$ & Measurement Items \\
\hline $\begin{array}{l}\text { Confirma- } \\
\text { tion [2] }\end{array}$ & $\begin{array}{l}\text { My expectation of using the NFriendConnector was } \\
\text { better than what I had expected. } \\
\text { The functionality provided by NFriendConnector was } \\
\text { better than what I had expected. } \\
\text { Overall, most of my expectations of using the } \\
\text { NFriendConnector were confirmed. }\end{array}$ \\
\hline $\begin{array}{l}\text { Perceived } \\
\text { Usefulness } \\
{[5]}\end{array}$ & $\begin{array}{l}\text { Using NFriendConnector would improve my } \\
\text { performance in establishing Facebook / [online social } \\
\text { network] connections with friends and acquaintances. } \\
\text { Using NFriendConnector would enhance my } \\
\text { effectiveness in establishing Facebook / [online social } \\
\text { network] connections. } \\
\text { Using NFriendConnector would increase my } \\
\text { productivity in managing my Facebook / [online social } \\
\text { network] connections. } \\
\text { I find NFriendConnector to be useful for establishing } \\
\text { Facebook / [online social network] connections. }\end{array}$ \\
\hline $\begin{array}{l}\text { Intention to } \\
\text { Use [5] }\end{array}$ & $\begin{array}{l}\text { Assuming that I have access to the NFriendConnector, I } \\
\text { intend to use it. } \\
\text { Given that the NFriendConnector is available to me, I } \\
\text { predict that I will use it. }\end{array}$ \\
\hline $\begin{array}{l}\text { Satisfaction } \\
\text { [2] }\end{array}$ & $\begin{array}{l}\text { How do you feel about your overall experience with the } \\
\text { NFriendConnector use? }\end{array}$ \\
\hline
\end{tabular}

No time limits were imposed on the subjects, as the main purpose of the experiment was to allow them to get a better understanding of the prototype, and explore its features in addition to performing the task of establishing a Facebook connection with their experimental partner. Most subjects were found to spend about 15 minutes evaluating the prototype.

\subsection{Data Analysis}

Table 3 reports the demographic details of the experimental sample. A total of 30 subjects participated in the experiment $(\mathrm{N}=$ 30). Being a Facebook user was not a pre-condition to participate in the experiment and the data indicates that the sample consisted of equal number of Facebook users and non users. However, subjects were asked whether they had experience with other social networking sites, and the data indicated that all subjects were experienced in online social networking. Subjects were mostly in the age group of 25 to 34, and most of them were pursuing a Master's degree in the university. Since subjects were volunteers who opted to participate, and we did not try to attain a balance between the genders, the sample turned out to be mostly male.

For validating the hypotheses, we tested the different regression models using SPSS. Summated scales were created for each variable. The primary objective of this experiment is to assess the extent to which subjects would consider adopting and using the prototype if it was made available to them. Accordingly, the first regression model tested the relationship between Perceived Usefulness and Intention to Use, and Satisfaction and Intention to Use. Table 4 reports the results of the regression analysis.
The data indicated that the regression model (model 1) was significant and both perceived usefulness and satisfaction were significantly associated with intention to use the prototype, thus supporting hypotheses $\mathrm{H} 4$ and $\mathrm{H} 5$. Overall, these two variables explained about $45 \%$ of the variance in the intention to use. Model 2 tested the relationship between satisfaction and its antecedents. Both perceived usefulness and confirmation were found to be significant predictors of satisfaction, thus supporting $\mathrm{H} 3$ and $\mathrm{H} 1$ respectively. Finally, model 3 indicates that the hypothesized relationship between confirmation and perceived usefulness (H2) is not supported.

Table 3: Sample Demographics

\begin{tabular}{|l|l|l|}
\hline \multicolumn{1}{|c|}{$\begin{array}{c}\text { Demographic } \\
\text { Variable }\end{array}$} & \multicolumn{1}{|c|}{ Categories } & Frequency (N=30) \\
\hline Age & Less than 25 & $4(13.3 \%)$ \\
& $25-34$ & $25(83.3 \%)$ \\
& Greater than 35 & $(3.3 \%)$ \\
\hline Gender & Female & $4(13.3 \%)$ \\
& Male & $26(86.7 \%)$ \\
\hline Educational Status & Bachelor's & $4(13.3 \%)$ \\
& Master's & $16(53.3 \%)$ \\
& PhD & $5(16.7 \%)$ \\
& Not specified & $5(16.7 \%)$ \\
\hline Facebook User & Yes & $15(50 \%)$ \\
& No & $15(50 \%)$ \\
\hline User of other Social & Yes & $29(96.7 \%)$ \\
Networking Site & No & $1(0.03 \%)$ \\
\hline
\end{tabular}

In addition to testing the hypothesized relationships, we controlled for several extraneous factors that can affect the findings. It was found that the demographic variables such as gender, age and educational background did not affect the responses. Since our experimental sample had equal number of Facebook users and non-users, we also tested whether this affected any of the variables of interest. Subjects Facebook usage status did not affect confirmation, perceived usefulness or their satisfaction with the NFriendConnector prototype application. However, whether or not a person was a Facebook user was significantly associated with their intention to use the prototype application ( $\mathrm{p}=0.043)$, with Facebook users reporting a higher intention to use the prototype (mean=3.7, s.d=0.82) than Facebook non-users $($ mean=2.9, s.d=1.21). The implications of these findings are discussed below.

Table 4: Regression Results

\begin{tabular}{|l|l|l|l|}
\hline Variables & $\begin{array}{l}\text { Standardized } \\
\text { Coefficients } \mathbf{B}\end{array}$ & Significance & Hypotheses \\
\hline \multicolumn{4}{|c|}{ Model 1: Dependent Variable - Intention to Use } \\
\hline $\mathbf{R}^{2}=\mathbf{0 . 4 9 2}$; Adjusted $\mathbf{R}^{2}=\mathbf{0 . 4 5 5} ; \mathbf{F}=\mathbf{1 3 . 0 9 3}$; Sig = 0 .000 (N = 30) \\
\hline $\begin{array}{l}\text { Perceived } \\
\text { Usefulness }\end{array}$ & 0.364 & $0.048(\mathrm{t}=2.067)$ & H4 supported \\
\hline Satisfaction & 0.413 & $0.027(\mathrm{t}=2.341)$ & H5 supported \\
\hline \multicolumn{4}{|c|}{ Model 2: Dependent Variable - Satisfaction } \\
\hline R2 = 0.506; Adjusted R2 = 0.469; F = 13.809; Sig = 0 .000 (N = 30) \\
\hline $\begin{array}{l}\text { Perceived } \\
\text { Usefulness }\end{array}$ & 0.560 & $0.000(\mathrm{t}=4.049)$ & H3 supported \\
\hline
\end{tabular}




\begin{tabular}{|l|l|l|l|}
\hline Confirmation & 0.339 & $0.021(\mathrm{t}=2.456)$ & H1 supported \\
\hline \multicolumn{4}{|c|}{ Model 2: Dependent Variable - Perceived Usefulness } \\
\hline R2 = 0.041; Adjusted R2 = 0.007; F = 1.208; Sig = 0 .281 (N = 30) \\
\hline Confirmation & 0.203 & $0.281(\mathrm{t}=1.099)$ & $\begin{array}{l}\text { H2 not } \\
\text { supported }\end{array}$ \\
\hline
\end{tabular}

\subsection{Findings and Implications}

A prototype design exercise can be considered successful when the target group of users find the application useful and are willing to use it. Our results suggest that subjects perceived the NFriendConnector as a useful application. Satisfaction and Perceived Usefulness were found to be significantly associated with users' intention to use the prototype. Satisfaction was also found to be significantly associated with perceived usefulness and confirmation or users' expectation. In addition to validating the usefulness of the NFriendConnector prototype, this study gives empirical evidence to the applicability of the expectationconfirmation model in the context of information system adoption and use. In line with the findings of Bhattacherjee [2], the experimental results indicate that Satisfaction was a stronger predictor of Intention to Use than Perceived Usefulness. Since post-use Satisfaction is grounded in users' first-hand experience with the system, it is believed to be more realistic, unbiased, and less susceptible to change, while Perceived Usefulness could be based solely on cognitive beliefs formed via second-hand information received from various sources.

The NFriendConnector is an innovative application that extends Facebook to its users' real worlds by allowing them to establish Facebook connections and access other Facebook features using their mobile phones. This can be a possible reason behind the insignificant relationship between confirmation and perceived usefulness. Since subjects encounter the application for the first time during the study, it is unlikely that they had pre-formed notions regarding its usefulness and what to expect from it. Accordingly, there was no expectation based confirmation that influenced users' perception regarding the usefulness of the prototype, therefore the insignificant relationship.

Even people who did not have prior experience with Facebook perceived the prototype as useful and were equally satisfied with it (reflected by no significant difference in the mean values of perceived usefulness or satisfaction for the user and non-user groups). This finding has important implications to both researchers and practitioners because it indicates that the current prototype is considered useful by users of other online social networking sites. Therefore, although this prototype was developed predominantly with the usage patterns of Facebook users in mind, the functionality provided by the NFriendConnector is considered meaningful and useful by users of other online social networking sites as well. However, we found that intention to use was significantly associated with whether or not a person was a Facebook user. This is possibly because Facebook non-users felt that the prototype was unlikely to be available to them for use, and therefore they reported lower intentions to use it.

Research and practical developments in the area of online social networking reflect a shift in the behavioral patterns and intentions of users of online social networks to reflect a tighter interlinking between socializing in real lives and the use of SNS. However, there have been few theoretically grounded studies which examine this changing usage pattern, and how providing applications and features to support this pattern, enhances satisfactions and intention to use the system. Our study addresses this gap by examining the use of the NFriendConnector prototype using the ECM as the theoretical framework, and provides empirical validation to the usefulness of such applications and features in the context of the most popular SNS - Facebook.

\section{LIMITATIONS}

The NFriendConnector prototype application, as well as this study should be interpreted in the context of its limitations. The NFriendConnector prototype demonstrates the extension of online social networking into the real world by integrating the Facebook platform with NFC-enabled mobile devices to provide a real time and ubiquitous access to online social networking. Therefore, the prototype supports only a limited set of Facebook functionality. Moreover, the prototype requires NFC-enabled mobile phones, which might prevent many users from adopting it.

This prototype was developed based on Facebook usage patterns, indicating that for Facebook users the direction of social networking is often from offline to online. This may raise issues regarding the generalizability of the prototype in other social networking platforms. In future research endeavors, experiments can be conducted to assess the perceptions of a different category or users using other social networking platforms (e.g. LinkedIn, Friendster, MySpace).

Conducting a laboratory experiment gives rise to limitations that are inherent to this research methodology. Future studies could assess usability in more natural social settings where users are less likely to feel constrained by an experimental setup. Studies that allow users to use and evaluate the prototype over a longer period of time could be designed to get a better gauge of their feelings and experiences with the prototype. Finally, we used university graduates and student subjects, which might have had some effect on the final results of the experiment. However, this does not really pose a big problem because our sample is generally reflective of the online social networking user profile and most subjects were not just Facebook users but also experienced in using other online social networks.

\section{CONCULSION AND FUTURE RESEARCH}

Current trends in social media, and mobile applications and devices indicate that there is an increasing demand for ubiquitous access to online social networks, and make it an integral part of people's real lives. In spite of this growing popularity of online social networking, currently, there is little connection between socializing in the real world and online social networking although users often use online social networking platforms like Facebook to map their actual offline contacts. Identifying this gap, we conceptualize, develop and evaluate the NFriendConnector prototype for to enable seamless social networking by extending online social networking into the real world using mobile devices.

The NFriendConnector allows users to make Facebook connections and also access certain other Facebook features, using NFC-enabled mobile phones. Further, social interactions can be 
enriched by complementing them with information from their online Facebook profiles to discover mutually shared interests. The experimental evaluation suggests that prospective users perceive the prototype as useful and are satisfied with its functionalities. Further, they indicate an intention to use the prototype if it is available to them. This provides empirical validity to our proposition regarding the importance of providing seamless social networking.

Our experimental results further indicate that even users who do not use Facebook perceive the prototype as useful and are satisfied with it. This indicates that there is a significant potential for developing similar prototypes for other online networking sites. For instance, being able to exchange contact information and establish connections may be particularly useful for online networking sites such as LinkedIn, which is a predominantly professional network. The functionality provided by the NFriendConnector can be thought of as the exchange of "online business cards", where NFC-enabled mobile phones allow users to exchange their contact information and professional interests even without having a mobile Internet connection. Future research could therefore support similar functionalities for other online social networks that are targeted towards different user groups. Finally, more comprehensive, long-term studies should be designed and executed to assess the extent to which different groups of users find the application useful and how its usage influences their online and offline social networking behavior.

\section{ACKNOWLEDGMENTS}

The NFriendConnector prototype was developed in the context of the research project Mobil50+ (Innovative NFC- und IT-basierte Dienstleistungen für mobiles Leben und Aktivität der Generation $50+)$. Mobil50+ is funded by the German Federal Ministry of Education and Research (BMBF - FKZ: 01FC08046). It is a joint project of the Technische Universität München and various partners. For further information, see www.projekt-mobil50.de.

\section{REFERENCES}

[1] Beach, A., M. Gartrell, S. Akkala, J. Elston, J. Kelley, K. Nishimoto, B. Ray, S. Razgulin, K. Sundaresan, B. Surendar, M. Terada, and R. Han 2008. WhozThat? evolving an ecosystem for context-aware mobile social networks. Network, IEEE. 22, 4 (2008) 50-55. DOI= http://dx.doi.org/10.1109/MNET.2008.4579771

[2] Bhattacherjee, A. 2001. Understanding Information Systems Continuance: An Expectation-Confirmation Model. MIS Quarterly. 25, 3 (2001) 351-370. DOI= http://dx.doi.org/10.2307/3250921

[3] Bramson-Boudreau, E. and L. Arathoon 2008. Analyst Insight: By the end of 2012, 950m user will be accessing social networking sites via mobile devices. 2008; Available from:

http://www.pyramidresearch.com/documents/02.21.08_AI_ Mobile\%20SNS.pdf.

[4] Cummings, J.N., B. Butler, and R. Kraut 2002. The quality of online social relationships. Commun. ACM. 45, 7 (2002) 103-108. DOI= http://dx.doi.org/10.1145/514236.514242

[5] Davis, F.D., R.P. Bagozzi, and P.R. Warshaw 1989. User acceptance of computer technology: a comparison of two theoretical models. Manage. Sci. 35, 8 (1989) 982-1003. DOI= http://dx.doi.org/10.1287/mnsc.35.8.982

[6] Eagle, N. and A. Pentland 2005. Social Serendipity: Mobilizing Social Software. IEEE Pervasive Computing. 4, 2 (2005) 28-34. DOI= http://dx.doi.org/10.1109/MPRV.2005.37

[7] Fogg, B.J. 2003. Persuasive Technology - Using Computers to Change What We Think and Do. Morgan Kaufman, San Francisco, CA.

[8] Karahanna, E., D.W. Straub, and N.L. Chervany 1999. Information technology adoption across time: a crosssectional comparison of pre-adoption and post-adoption beliefs. MIS Q. 23, 2 (1999) 183-213. DOI= http://dx.doi.org/10.2307/249751

[9] Kostakos, V. and E. O’Neill 2008. Cityware: Urban computing to bridge online and real-world social networks. In Handbook of Research on Urban Informatics: the Practice and Promise of the Real-Time City, M. Foth, Ed. Information Science Reference, IGI Global, 196-205.

[10] Lampe, C., N. Ellison, and C. Steinfield 2006. A face(book) in the crowd: social Searching vs. social browsing. In Proc. 20th anniv. conf. on CSCW. 2006. Banff, Alberta, Canada: ACM. DOI= http://dx.doi.org/10.1145/1180875.1180901

[11] Lampe, C.A.C., N. Ellison, and C. Steinfield 2007. A familiar face(book): profile elements as signals in an online social network. In Proc. CHI. 2007. San Jose, California, USA: ACM. DOI= http://dx.doi.org/10.1145/1240624.1240695

[12] Leimeister, J.M. and H. Krcmar 2006. CommunityEngineering Systematischer Aufbau und Betrieb Virtueller Communitys im Gesundheitswesen. WIRTSCHAFTSINFORMATIK. 48, 6 (2006) 418-429. DOI= http://dx.doi.org/10.1007/s11576-006-0094-y

[13] Leimeister, J.M., K. Schweizer, S. Leimeister, and H. Krcmar 2008. Do virtual communities matter for the social support of patients?: Antecedents and effects of virtual relationships in online communities. Information Technology \& People. 21, 4 (2008) 350 - 374. DOI= http://dx.doi.org/10.1108/09593840810919671

[14] Lin, C.S., S. Wu, and R.J. Tsai 2005. Integrating perceived playfulness into expectation-confirmation model for web portal context. Inf. Manage. 42, 5 (2005) 683-693. DOI= http://dx.doi.org/10.1016/j.im.2004.04.003

[15] Oliver, R.L. 1980. A cognitive model for the antecedents and consequences of satisfaction. Journal of Marketing Research. 17, 4 (1980) 460-469.

[16] Oliver, R.L. 1993. Cognitive, Affective, and Attribute Bases of the Satisfaction Response. Journal of Consumer Research: An Interdisciplinary Quarterly. 20, 3 (1993) 41830.

[17] Parks, M.R. and K. Floyd 1996. Making Friends in Cyberspace. Journal of Communication. 46, 1 (1996) 80-97. DOI= http://dx.doi.org/10.1111/j.1083-6101.1996.tb00176.x

[18] Parks, M.R. and L.D. Roberts 1998. 'Making Moosic': The Development of Personal Relationships on Line and a Comparison to their Off-Line Counterparts. Journal of 
Social and Personal Relationships. 15, 4 (1998) 517-537. DOI= http://dx.doi.org/10.1177/0265407598154005

[19] Persson, P., J. Blom, and Y. Jung 2005. DigiDress: A Field Trial of an Expressive Social Proximity Application. In UbiComp 2005: Ubiquitous Computing, 195-212. DOI= http://dx.doi.org/10.1007/11551201_12

[20] Persson, P. and Y. Jung 2005. Nokia sensor: from research to product. In Proc. DUX. 2005. San Francisco, California: AIGA: American Institute of Graphic Arts.

[21] Pietiläinen, A.-K., E. Oliver, J. Lebrun, G. Varghese, and C. Diot 2009. MobiClique: Middleware for Mobile Social Networking. In Proc. Sec. ACM SIGCOMM Workshop on Online Social Networks. 2009. Barcelona, Spain: ACM. DOI= http://dx.doi.org/10.1145/1592665.1592678
[22] Richter, A., M. Koch, and J. Krisch 2007. Social Commerce Eine Analyse des Wandels im E-Commerce.

[23] Spreng, R.A., S.B. MacKenzie, and R.W. Olshavsky 1996. A Reexamination of the Determinants of Consumer Satisfaction. The Journal of Marketing. 60, 3 (1996) 15-32. DOI= http://dx.doi.org/10.2307/1251839

[24] Ziv, N.D. and B. Mulloth 2006. An Exploration on Mobile Social Networking: Dodgeball as a Case in Point. In Proc. of the ICMB. 2006: IEEE Computer Society. DOI= http://dx.doi.org/10.1109/ICMB.2006.8 